\title{
Influence of System Performance on Layer Thickness Determination Using Terahertz Time-Domain Spectroscopy
}

\author{
Stefan Weber ${ }^{1,2}$ iD $\cdot$ Lukas Liebelt $^{3}$ - Jens Klier ${ }^{1} \cdot$ Tobias Pfeiffer $^{1} \cdot$ Daniel Molter $^{1}$. \\ Frank Ellrich ${ }^{3}$ - Joachim Jonuscheit ${ }^{1} \cdot$ Georg Von Freymann ${ }^{1,2}$
}

Received: 26 June 2019 / Accepted: 15 January 2020 / Published online: 28 January 2020

(C) The Author(s) 2020

\begin{abstract}
The quality of coatings in industrial applications and scientific research with thicknesses in the micrometer range is an important criterion for quality management. Therefore, thickness determination devices are of high interest. Terahertz time-domain spectroscopy systems have demonstrated the capability to address thickness determination of dielectric single- and multilayer coatings on different substrates. However, due to the large range of different samples, there are different performance requirements to ensure a high-quality determination result. In this paper, we investigate the influence of system parameters - bandwidth and dynamic range - on thickness determination performance for a single-layer coating on metal substrates with thicknesses from 0.5 to $100 \mathrm{pm}$, based on measurements and numerical calculations within dynamic ranges from 10 to $90 \mathrm{~dB}$ and bandwidths from 1.5 to $10 \mathrm{THz}$.
\end{abstract}

Keywords Terahertz time-domain spectroscopy · Thin films · Thickness determination · Industrial · Application

\section{Introduction}

The measurement of layer thicknesses is an important step in the quality management process in many different industrial fields. Thereby, the product spectrum ranges from multilayered foils with thicknesses of about $1 \mathrm{pm}$ per layer, over automotive coatings from few micrometers

Stefan Weber

stefan.weber@itwm.fraunhofer.de

1 Fraunhofer Institute for Industrial Mathematics ITWM, Center for Materials Characterization and Testing, 67663 Kaiserslautern, Germany

2 Department of Physics and Research Center OPTIMAS, Technische Universität Kaiserslautern, 67663 Kaiserslautern, Germany

3 University of Applied Sciences, TH Bingen, 55411 Bingen, Germany 
to several tens of micrometers to five layer plastic tubes with a thickness of several hundreds of micrometers.

Recently, terahertz time-domain spectroscopy (TDS) approaches have proven to be a promising new technology to address the demand of contactless thickness measurement devices [1-7]. Due to the large range of different applications for thickness determination with TDS systems, different requirements have to be fulfilled. Besides the handling of environmental influences, temperature, humidity stability, and vibration influences [8,9], the principle performance of the measurement device is a crucial factor: "Which performance bandwidth and dynamic range - is necessary to reproducibly determine the properties of the samples of interest?" Often, there is a trade-off between these characteristics and measurement speed. Therefore, the knowledge of the necessary performance is helpful to optimize the measurement process.

In this paper, we experimentally and numerically investigate the influence of bandwidth (BW) and dynamic range (DR) on the thickness determination performance of single-layer coatings on a metal substrate. Therefore, we vary the performance of the used TDS system in BW from 1.5 to 4.5 THz and in maximum DR from 22 to $63 \mathrm{~dB}$ to measure different thicknesses of a typical automotive basecoat. With numerical calculations, the measured results are confirmed; therefore, the developed numerical approach based on the Drude model can be used too.

\section{Terahertz Time-Domain Spectroscopy and Layer Thickness Determination}

For thickness determination of thin layers in the terahertz frequency range, a pulsed terahertz radiation, usually generated with photoconductive switches, is used. In the left part of Fig. 1, the TDS approach is schematically depicted. Femtosecond laser pulses, generated by a laser source, are split via a beam splitter and guided to a terahertz emitter and detector, respectively. In order to implement the pump-probe concept of a TDS system, a delay line with a fast shaking mirror is used to vary a temporal delay between the laser pulse to the emitter and detector. As emitter and detector antennas, GaAs-based photoconductive switches are used [10]. A more detailed explanation can be found in [11].

The generation of a terahertz pulse using a photoconductive switch can theoretically be described by the Drude model. From Maxwell's equations, the emitted electromagnetic field $E_{\mathrm{THz}}$ depends on the change of the current density $J: E_{\mathrm{THz}} \propto \partial J / \partial t$ with $J=$ env, where $e$ is the elementary charge, $n$ is the charge carrier density, and $v$ the speed of charge carriers. Therefore, $\partial J / \partial t$ can be written as follows:

$$
\frac{\partial J}{\partial t}=e v \frac{\partial n}{\partial t}+e n \frac{\partial v}{\partial t}
$$

The temporal change of the charge carrier density is described by [12]:

$$
\frac{d n(t)}{d t}=-\frac{n}{\tau_{c}}+G=-\frac{n}{\tau_{c}}+n_{0} \cdot \exp \left(-t^{2} / \delta t^{2}\right),
$$

with $G \propto I$ being the carrier generation rate proportional to the laser intensity $I, \delta t$ being the laser pulse duration, and $\tau_{c}$ being the carrier trapping time. With Eq. (2), the electromagnetic field can be formulated as follows: 


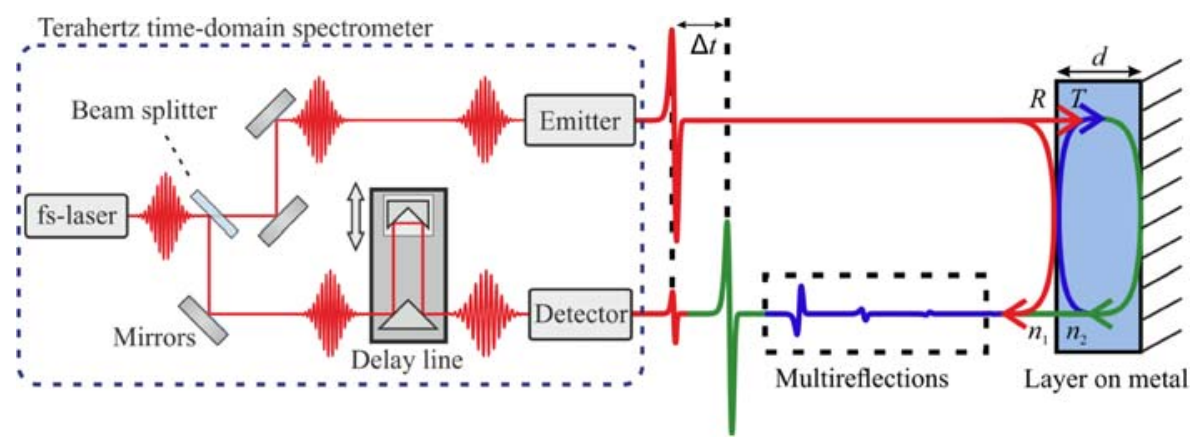

Fig. 1 Terahertz TDS setup with a femtosecond laser pulse source and semiconductor photoconductive switches as emitter and detector. With a delay line in one arm, the pump-probe concept is realized. Defined by the Fresnel equations, the emitted terahertz pulse is partly reflected and transmitted at the first interface between air and dielectric layer. At the back side, between layer and metal substrate, the pulse is completely reflected which is also covered by Fresnel equations predict the behavior for other samples and performances. With the numerical calculations, we cover a thickness range from 0.5 to $100 \mu \mathrm{m}$ over a large range of available $\mathrm{BW}(1.5 \mathrm{THz}$ to $10 \mathrm{THz})$ and maximum dynamic ranges (10 dB to $90 \mathrm{~dB})$.

$$
E_{\mathrm{THz}} \propto \frac{\partial J}{\partial t}=\mathrm{ev} \frac{\partial n}{\partial t}+\mathrm{en} \frac{\partial v}{\partial t} \approx \mathrm{ev} \frac{\partial n}{\partial t}=-\frac{n}{\tau_{c}}+G
$$

It is worth noting that the radiation emitted by the acceleration of the carriers is negligible compared to the effect the change in density has, and hence is not discussed in further detail [12]. In Fig. 2, the formalism for describing the terahertz pulse generation is illustrated. The carrier density (red) increases while the laser pulse $G(t)$ (blue) illuminates the semiconductor and decays afterwards. The carrier density $n(t)$ is temporally delayed to the laser pulse with the maximum at the zero-crossing point of the field $E(t)$ (green).

Thickness determination With the generated terahertz pulses, the layer thickness determination can be performed, illustrated in the right part of Fig. 1. In general, terahertz radiation is reflected by conductive materials, e.g., metal- or carbon-reinforced plastic, but penetrates

Fig. 2 The intensity of the laser pulse $G(t)$ (blue), the resulting carrier density $n(t)(\mathrm{red})$, and the emitted terahertz pulse $E(t)$ (green) are shown. The density reaches its maximum when carrier trapping matchs the generation rate

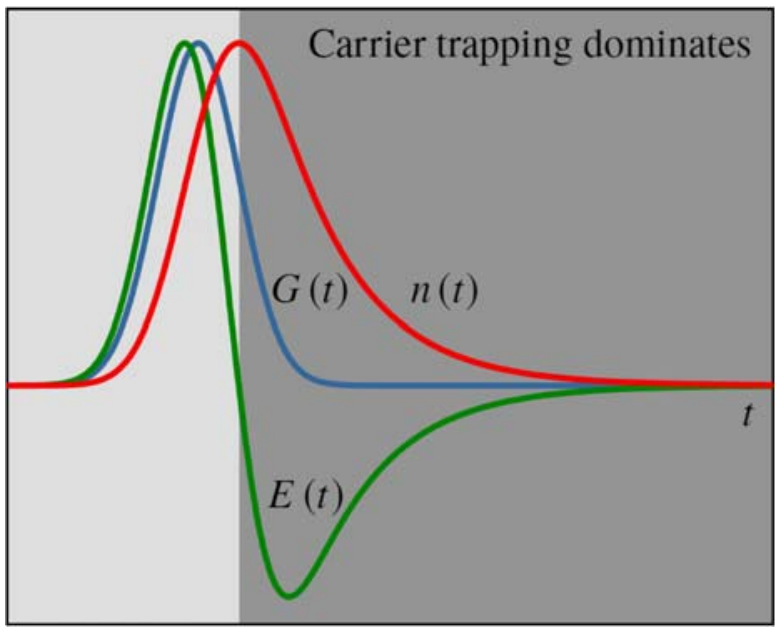


dielectric materials, e.g., paint, plastic, and ceramic. Defined by the Fresnel equation, the reflectance $R$, which defines the ratio between incident and reflected intensity $I_{r}(t)=R$. $I(t)$ with $0<R<1$, can be calculated from the amplitude reflection coefficient $R=r \frac{1}{1,2}$, with the coefficient under perpendicular incidence:

$$
r_{1,2}=\frac{n_{1}-n_{2}}{n_{1}+n_{2}} .
$$

$n_{1 ; 2}=\widetilde{n}_{1,2}+i \kappa_{1,2}$ are the complex refractive indices of the involved layers. Furthermore, the transmitted part of the wave with wavelength $\lambda_{0}$ is damped with

$$
E(d)=E_{0} \exp \left(-\frac{2 \pi \kappa d}{\lambda_{0}}\right) .
$$

Depending on the optical thickness of the layer of interest $d_{\text {opt }}=n \times d_{\text {mech }}$, a temporal delay or, in other words, a phase shift is induced. Measuring the completely reflected waveform, the thickness of the layer can be calculated with knowledge of the temporal delay $\Delta t$ and the refractive index of the layer. For thin layers with optical thicknesses of $d_{\mathrm{opt}}<150 \mu \mathrm{m}$, the pulses from the front and back interface are not temporally separable anymore. However, in order to determine the thicknesses of thin, single, as well as multilayer samples, a transfer-matrix model-based algorithm can be used to extract the thickness information from the measured waveform [5, 13]. In [14], an optimized transfer-matrix model, Rouard-based approach, is described for thickness determination of up to four layers on different substrates. With the Rouard model, the influence of a layered sample on an incoming terahertz pulse can be iteratively calculated. This resulting pulse is compared with the measured one, and by varying the input thicknesses, the best match between calculated and measured pulse can be found; hence, the thickness of all layers can be determined. In order to find the best parameter set for fitting the calculated pulse to the measured pulse, i.e., finding the global minimum in the parameter space, different optimization approaches, e.g., stratified dispersive model, analytically solving the transfer-matrix equations for a certain number of layers or different evolutionary algorithms can be used $[13,15,16]$.

Bandwidth-dynamic range-signal-to-noise ratio The performance of a terahertz TDS system is defined by the achievable BW, the DR, and the signal-to-noise ratio (SNR) mainly described by the maximum values, respectively. The DR and SNR can be defined in the time and frequency domain as in [17]:

$$
\text { SNR }=\frac{\text { mean magnitude of amplitude }}{\text { standard deviation of amplitude }}
$$

and

$$
D R=\frac{\text { maximum magnitude of amplitude }}{\text { rms of noise floor }}
$$

In Fig. 3 (left), the normalized spectral amplitudes of 100-THz pulses, measured with an integration time of $1 \mathrm{~s}$, are shown. Due to cross-talk between emitter and detector, the noise level is typically frequency-dependent. Therefore, it is not sufficient to take the noise level at high frequencies to calculate the dynamic range. However, in the case of a systematic crosstalk, this background can be easily subtracted from the signal measurement for a certain delay line position; therefore, it does not influence the DR. 

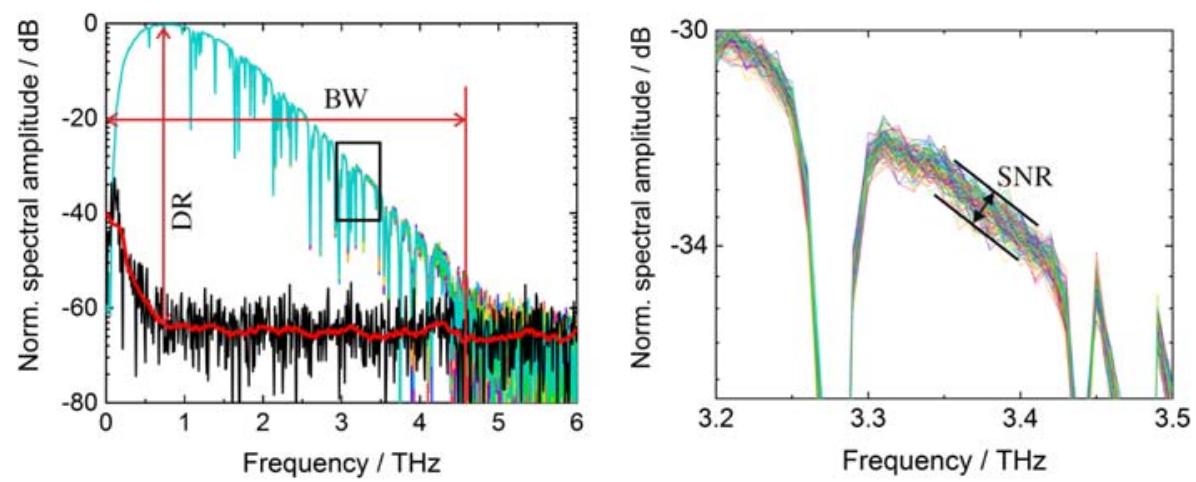

Fig. 3 (left) Spectral amplitude of 100 pulses, measured with a measurement time of $1 \mathrm{~s}$ at an uncoated metal plate. The frequency-dependent noise amplitude (black) is measured without a sample. The maximum dynamic range is defined as the difference between noise level (red) and signal amplitude. (right) Zoomed spectra to illustrate the SNR of a terahertz TDS system

The BW of a measured terahertz pulse is defined as the frequency, where the signal amplitude is not distinguishable from the noise amplitude anymore. In the presented measurements, the bandwidth is $4.5 \mathrm{THz}$ with a maximum dynamic range of $\mathrm{DR}=61: 8 \mathrm{~dB}$, calculated in the frequency domain.

Both parameters can be calculated with only one reference (metal sample) and one noise (without sample) measurement. For SNR, multiple reference measurements have to be done to obtain the mean and standard deviation of the magnitude of amplitude. In Fig. 3 (right), the varying amplitude of 100 measurements between 3.2 and $3.5 \mathrm{THz}$ is illustrated. For a measurement time of $1 \mathrm{~s}$, the maximum SNR of the used device is SNR $=65: 1 \mathrm{~dB}$. For thickness determination, these three parameters directly influence the determination performance, described by accuracy, reproducibility, and thickness range. In order to assess these performance values, the standard deviation and for thinner layers an acceptance range is introduced. Both parameters are well-suited to describe the accuracy and reproducibility of the determination performance. The thickness range is given by an application defined threshold for the achieved standard deviation and acceptance range values.

In the following sections, the influence of these parameters on the thickness determination of a single-layer coating is investigated experimentally and numerically.
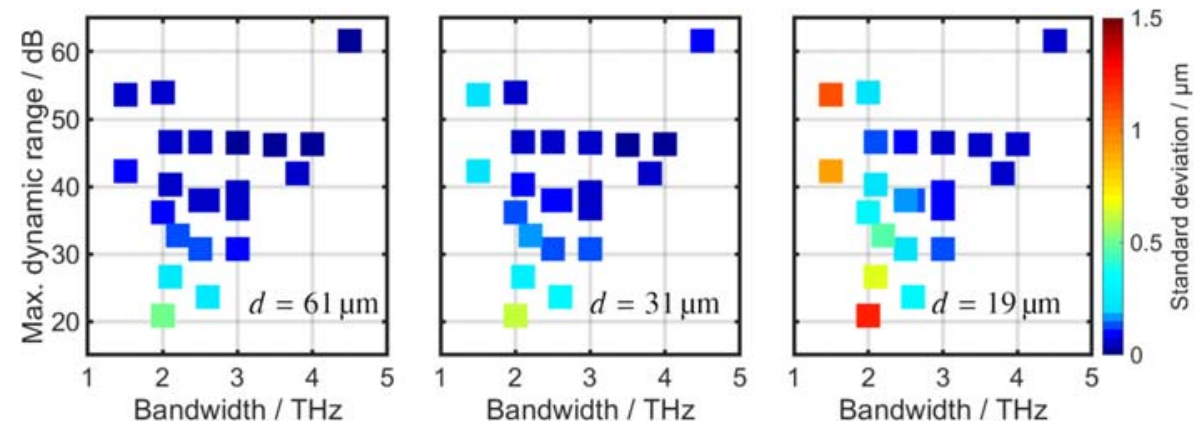

Fig. 4 Standard deviation for 22 combinations of bandwidth and dynamic range. For each sample, the standard deviation increases with lower bandwidth and dynamic range. The thinner the layer the higher the standard deviation for each performance combination 


\section{Measurement Setup and Results}

In order to experimentally investigate the influence of dynamic range and bandwidth, three different single-layer samples on polished metal substrates with the same coating but different thicknesses are measured with a fiber-coupled terahertz TDS system. The photoconductive emitter and detector are placed in a measurement head with reflection geometry, connected with the supply unit via 5-m optical and electrical cables. The detector signal is amplified by an amplifier with a noise current of $135 \mathrm{fA} / \sqrt{\mathrm{Hz}}$ and a linearity of $<1 \%$. The amplifier is one of the most important noise sources in a TDS system.

With this device, we achieve a maximum dynamic range of $63 \mathrm{~dB}$ and a bandwidth of 4.5 THz with an integration time of $2 \mathrm{~s}$. In order to cover a large range of performance values, different adjusting screws are available: reducing the integration time down to $25 \mathrm{~ms}$, misaligning the working distance between measurement head and samples of about $1 \mathrm{~mm}$, and increasing the laser pulse duration using different fiber lengths to induce dispersion effects. With these adjusting screws, we are able to vary the performance to 22 different BW-DR configurations. By varying these parameters, the bandwidth as well as the DNR, are influenced simultaneously. It is worth noting that the spectral shape is slightly influenced by varying the fiber lengths and the working distance. However, within the 22 combinations, there are no crucial dips in the spectra and this effect can be neglected.

The metal substrates are painted with a single-layer automotive coating with a homogeneous complex refractive index of $n=1.620+i .0 .030$ and thicknesses of $d=61 \mu \mathrm{m}, d=$ $31 \mu \mathrm{m}$, and $d=19 \mu \mathrm{m}$, respectively. For calculating the thicknesses of the coatings, the algorithm, described in section 2 with a time consumption of less than $1 \mathrm{~ms}$ per measurement, is used. For each configuration and sample, 1000 measurements are done to calculate the standard deviation of the thickness results. It is worth noting that the standard deviation of the thickness calculation algorithm is more than two orders of magnitude smaller than the standard deviation for multiple measurements with the best performance.

The standard deviations of 1000 thickness measurements for the 22 combinations are shown in Fig. 4. For all samples, the standard deviation increases with lower BW and DR. However, the influence is larger for thinner layers: for a BW of only $2 \mathrm{THz}$ with a DR of $20.8 \mathrm{~dB}$, the standard deviation is only $0.5 \mu \mathrm{m}$ for the thick layer with a thickness of $61 \mu \mathrm{m}$, but more than $1.2 \mu \mathrm{m}$ for the $19-\mu \mathrm{m}$ sample.

For these measurement data, only the DR and the available BW is analyzed. The SNR is, as well as the DR, mainly affected by the white noise and signal amplitude. A change of the SNR without changing the DR is only possible for a frequency-dependent emitternoise-limited TDS system, e.g., fluctuations in the laser power to the emitter module or coupling fluctuations in the delay line. In this case, it would be possible to change the signal amplitude and in the same way the noise amplitude to obtain the same DR but a different SNR. For all other influences - frequency-independent emitter fluctuations or not emitter-noise limited systems - both parameters, SNR and DR, are influenced in the same way. However, in general, TDS systems are typically detector-noise-limited devices; therefore, the DR and SNR cannot be independently manipulated. For maximum DR between 10 and $90 \mathrm{~dB}$, the maximum SNR is about $2.5 \mathrm{~dB}$ higher than the maximum DR, confirmed by measurements and calculations. Hence, the DR is used as evaluation criterion to cover both DR and SNR effects.

In order to investigate the influence of BW and DR in more detail, many samples and combinations have to be prepared and measured. To overcome this effort, we numerically 
reproduced the emitting characteristic of the emitter for different settings and calculated the layer influence on the terahertz pulses.

\section{Numerically Calculated Results}

Due to the limited parameter range in the presented experiments to investigate the influence of system performance on thickness determination, numerical calculations are a helpful tool to reduce the effort for large-scale studies. Based on the model, described in section 2, the characteristics of a terahertz TDS system can be numerically reproduced.

In order to evaluate the quality of the numerically calculations, the same BW and DR combinations were calculated. Then, 1000 different noisy pulses per combination are applied on the three samples with identical material parameters and thicknesses, and the standard deviations of the resulting thicknesses are compared with the measured results.

Figure 5 shows the calculated spectral characteristics after each step in the calculation process. First, an ideal terahertz pulse, based on Drude model, is calculated (blue). Then, the water absorption, as found in the HITRAN database [18] (red) and white noise (green) is added. This results in a realistic terahertz spectrum, which is comparable to a real spectrum. Here, the noise level is assumed as frequency-independent, in contrast to Fig. 3.

Based on Eq. (3), the emitted electric field depends mainly on the laser pulse duration $\delta \mathrm{t}$ and the trapping time of the carriers $\delta t$ as well as additional white noise:

$$
E_{T H z}=-\frac{n}{\tau_{c}}+n_{0} \cdot \exp \left(-t^{2} / \delta t^{2}\right) .
$$

With these parameters, the DR (influenced by the noise level) and the BW (influenced by the laser pulse duration) can be adjusted. Using the presented calculation model, the measurements can be numerically reproduced, as depicted in Fig. 6.

For each sample, the calculated results are in good agreement with the measured data: the standard deviation due to varying $\mathrm{BW}$ and DR shows the same behavior. The deviation increases with lower BW and DR, and the effect is stronger for thinner layers. Only for the

Fig. 5 Ideal calculated terahertz spectrum based on the Drude model (blue). With water vapor absorption (red) and white noise (green), a realistic terahertz spectrum can be generated

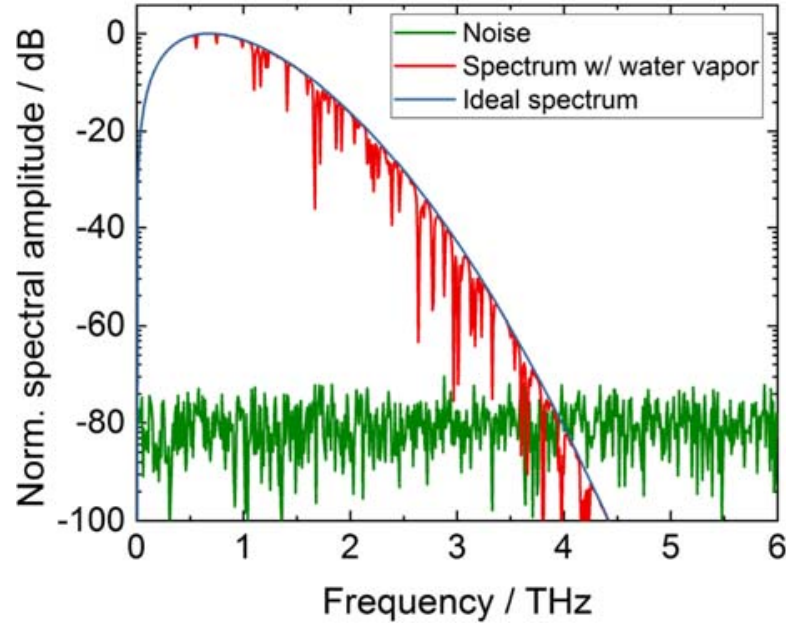



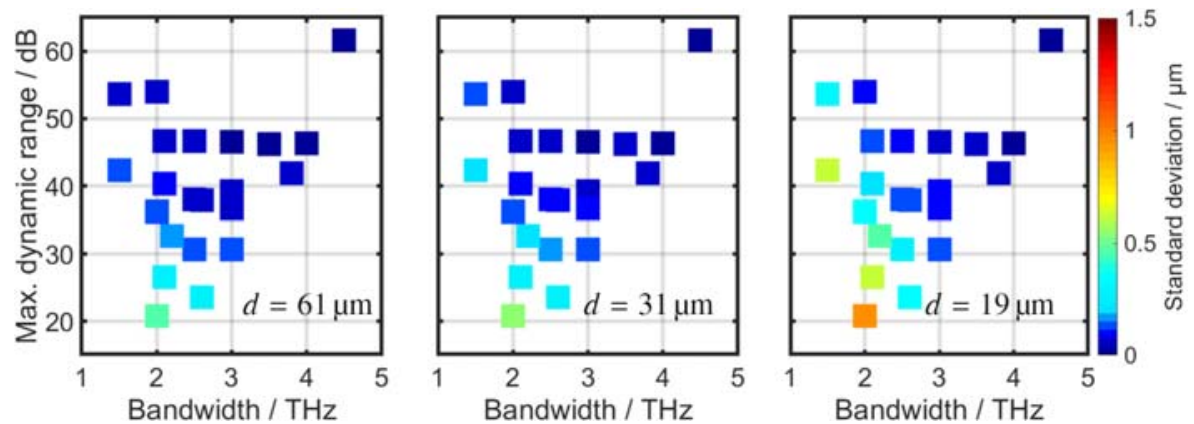

Fig. 6 Numerically, there is a calculated influence of varying BW and DR on thickness determination. The calculated standard deviations agree well with the measured data and show the same behavior for all samples

19- $\mu \mathrm{m}$ sample, the calculated values for the lowest bandwidth show a smaller deviation as the measured data.

The comparison confirms that the developed numerical tool is able to reproduce the measurement with real sample interaction. Therefore, we used the tool to investigate this behavior in more detail.

To do that, we calculated 17 different maximum DR values, from 10 to $90 \mathrm{~dB}$ and $18 \mathrm{BW}$ values from 1.5 to $10 \mathrm{THz}$, which results in 306 combinations. For each combination, 32 different thicknesses of a coating with $n=2.000+$ i. 0.000 are used to cover a wide thickness range from 0.5 to $100 \mu \mathrm{m}$. Therefore, $N=306.32 .1000=9.8$ million pulses are calculated and analyzed. Furthermore, to investigate the influence of different refractive indices (real and imaginary part), four different coatings are calculated. In total, 40 million pulses are used to provide sufficient statistic results with a wide range of combinations. In order to illustrate the range of investigated BW and DR values, Fig. 7 shows the time-domain (left) and frequencydomain signal (right) with the best $(10 \mathrm{THz}$ with $90 \mathrm{~dB})$ and worst $(1.5 \mathrm{THz}$ with $10 \mathrm{~dB})$ performance.

As an example, the results for $d=100 \mu \mathrm{m}, d=50 \mu \mathrm{m}, d=10 \mu \mathrm{m}$, and $d=5 \mu \mathrm{m}$ are depicted as $2 \mathrm{D}$ plots in Fig. 8 (left). The calculation with higher resolution in BW and DR shows qualitatively the same behavior as the measured data before. The error increases with thinner samples and lower performance.
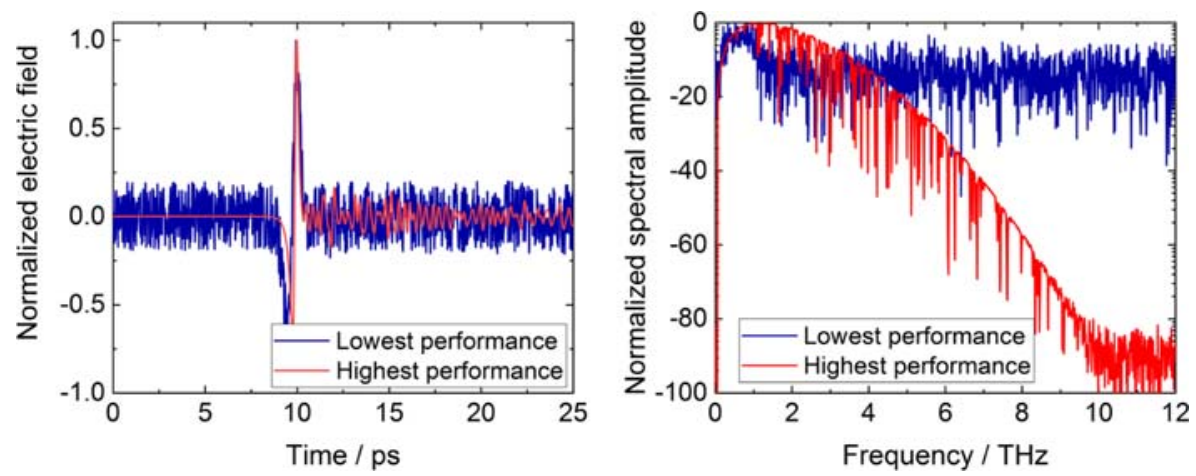

Fig. 7 For the calculation, the bandwidth is varied from 1.5 to $10 \mathrm{THz}$ and the maximum dynamic range from 10 to $90 \mathrm{~dB}$. The red lines show the highest performance and the blue lines the lowest performance in time domain (left) and frequency domain (right) 

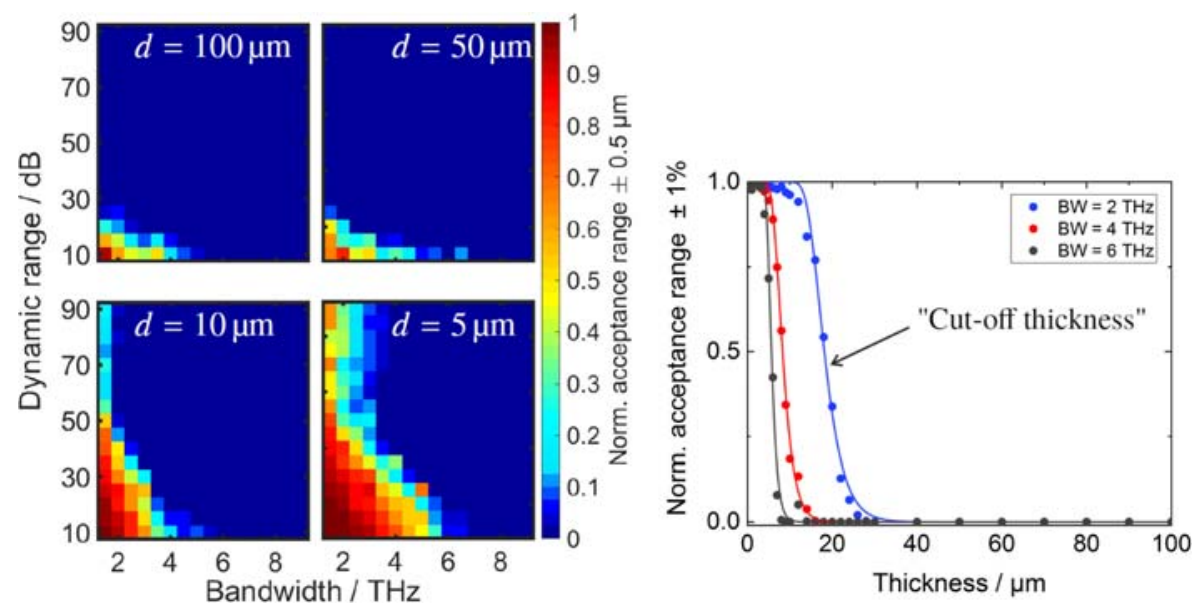

Fig. 8 (left) Normalized acceptance range of $\pm 0.5 \mu \mathrm{m}$ for four different samples: the thinner the layer the higher the error for a given performance. (right) Thickness dependent relative acceptance range of $1 \%$ for different bandwidths with a maximum dynamic range of $40 \mathrm{~dB}$. The "cut-off thickness" decreases with larger bandwidth

In particular, for thin layers (less than about $10 \mu \mathrm{m}$ ), the standard deviation is not a valid evaluation criterion anymore. In case of decreasing performance, the thickness results show lower deviations as results calculated with better performances, due to a strong accumulation at others, and therefore wrong thickness results. Hence, for further investigations, a normalized acceptance range is used to evaluate the results in order to take into account real thickness values. In Fig. 8 (left), an absolute acceptance range of $0.5 \mu \mathrm{m}$ is used as validation criterion: 1 means no thickness result is within the range and 0 means all results are within the range.

In order to evaluate which performance is necessary to address a certain thickness range with a desired deviation, the presentation in Fig. 8 (right) is more suitable. The acceptance range depending on the thickness is depicted for three different bandwidths with a maximum dynamic range of $40 \mathrm{~dB}$. With this, it is possible to define a bandwidth-dependent "cut-off thickness," where the error changes from high to low level.

It is worth noting that to cover the large thickness range from 0.5 to $100 \mu \mathrm{m}$, a relative acceptance range is a more suitable criterion, because already for $5-\mu \mathrm{m}$ thick layers, an
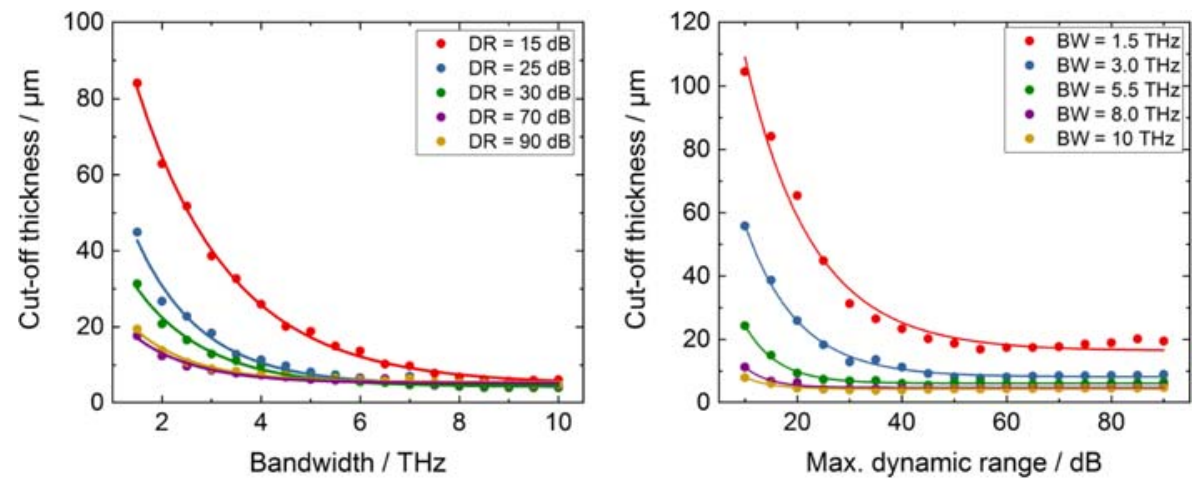

Fig. 9 (left) Bandwidth-dependent cut-off thickness for different maximum DR. For low bandwidths, the decrease of the thicknesses is clearly visible. However, for higher bandwidths, there is saturation effect. This behavior is also visible for the DR-dependent cut-off thicknesses (right) 

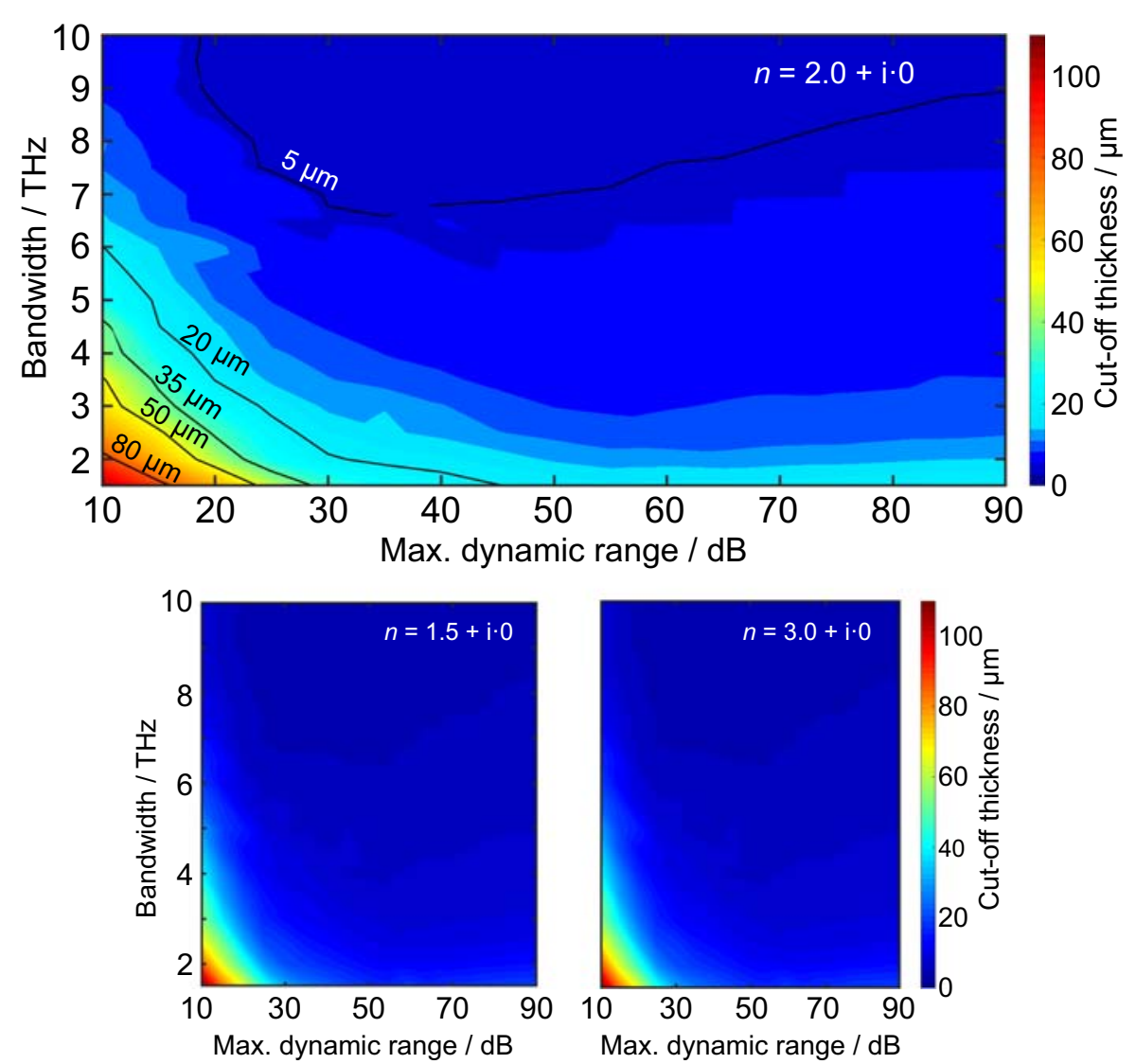

Fig. 10 (top) Cut-off thickness heat map for a single-layered sample. The cut-off thickness increases with decreasing bandwidth and dynamic range. (bottom) Results for two other coatings with the same behavior

absolute range of $0.5 \mu \mathrm{m}$ means a relative error of $10 \%$. Therefore, a normalized relative acceptance range of $1 \%$ will be used for the further discussions.

The cut-off thickness $d_{\text {cut }}$, fitted with a Gumbel-function $f(x)=1-\exp \left(-\operatorname{esp}\left(\frac{x-d_{\text {cut }}}{b}\right)\right)$, is calculated for all BW-DR combinations for a single-layered sample with $n=2.000+\mathrm{i} .0 .000$ to obtain the BW- and DR-dependent cut-off thickness, depicted in Fig. 9. The cut-off thickness shows an exponential decrease for both parameters with a saturation effect for high bandwidths and dynamic ranges at about $4 \mu \mathrm{m}$, respectively, using a relative acceptance range of $1 \%$, which means an absolute range of $\pm 40 \mathrm{~nm}$. The corresponding 2D heat map for the cut-off thickness is shown in Fig. 10 (top). For low maximum dynamic ranges of less than $20 \mathrm{~dB}$ and bandwidths of less than $3 \mathrm{THz}$, the cut-off thickness is about $50 \mu \mathrm{m}$, and therefore not sufficient for automotive coating thickness determination applications. For typical automotive coatings with thicknesses between 8 and $45 \mu \mathrm{m}$, a BW of more than $3 \mathrm{THz}$ with a maximum dynamic range of more than $55 \mathrm{~dB}$ is necessary. However, also a combination of $4 \mathrm{THz} \mathrm{BW}$ and only $35 \mathrm{~dB}$ DR can be used to obtain a cut-off thickness of about $8 \mu \mathrm{m}$. For thinner layers, less than $5 \mu \mathrm{m}$, a BW of more than $6.5 \mathrm{THz}$ is necessary to achieve a deviation of less than $50 \mathrm{~nm}$.

The calculated cut-off thicknesses for other layers show the same behavior. In Fig. 10 (bottom), the cut-off thicknesses for $n=1.5+\mathrm{i} .0$ (left) and $n=3.0+\mathrm{i} .0$ (right) is shown. There 
are no significant differences to the layer with $n=2.0+\mathrm{i} .0$. For higher imaginary parts of the refractive index, the thickness deviation increases with thicker layers, due to the high absorption, and therefore a weaker signal from the backside of the coating (not shown). In particular, for higher BW, the cut-off thickness increases with higher DR (dark blue area). This effect can be explained with other, not covered effects, which plays a more important role for thin layers. In general, the terahertz waveform is spline-interpolated for calculating the thicknesses. However, a spline interpolation adds noise and slightly deforms the measured signal. To overcome this influence, zero padding can help to reduce the noise level and to optimize the calculation quality. The investigation of the influences of different interpolation methods is part of future studies.

\section{Conclusions}

We experimentally and numerically investigated the influence of BW, DR, and SNR on the thickness determination performance of a single-layer coating on a metallic substrate, based on the terahertz TDS approach. In order to evaluate the thickness results, a normalized absolute and relative acceptance range is used as criterion to generate a meaningful statement over a large range of thicknesses. The measured data show an increasing number of errors with decreasing bandwidths and dynamic ranges. These results are confirmed with high quality by numeric calculations. Therefore, the developed numeric tool, using the Drude model and a Rouardbased thickness determination method, is used to investigate the behavior in more detail by calculating more than 40 Mio. terahertz pulses. As a result, a BW- and DR-dependent heat map with the achievable cut-off thickness, as a look-up table, is generated for different single-layered coatings. As an example, a BW of more than $3 \mathrm{THz}$ in combination with a maximal DR of more than $55 \mathrm{~dB}$ is necessary for a typical automotive coating. With the numeric tool, we are able to predict the behavior of single as well as multilayer samples. To cover a larger range of effects, other emitter-detector concepts, e.g., spintronic sources should also be investigated in the future. In case of emitter-noise-limited terahertz devices, the signal-to-noise influence is not negligible anymore, and therefore the investigation of this effect is of high interest.

Acknowledgements Open Access funding provided by Projekt DEAL.

Open Access This article is licensed under a Creative Commons Attribution 4.0 International License, which permits use, sharing, adaptation, distribution and reproduction in any medium or format, as long as you give appropriate credit to the original author(s) and the source, provide a link to the Creative Commons licence, and indicate if changes were made. The images or other third party material in this article are included in the article's Creative Commons licence, unless indicated otherwise in a credit line to the material. If material is not included in the article's Creative Commons licence and your intended use is not permitted by statutory regulation or exceeds the permitted use, you will need to obtain permission directly from the copyright holder. To view a copy of this licence, visit http://creativecommons.org/licenses/by/4.0/.

\section{References}

1. K. Su, YC. SShen, JA. Zeitler, "Terahertz sensor for non-contact thickness and quality measurement of automobile paints of varying complexity," IEEE Transactions on Terahertz Science and Technology 4 (4), 432-439 (2014). 
2. J.L.M. van Mechelen, D.J.H.C. Maas, and H. Merbold, "Paper sheet parameter determination using terahertz spectroscopy," Infrared, Millimeter, and Terahertz waves (IRMMW-THz), $201641 \mathrm{st}$ International Conference on (2016).

3. F. Ellrich, J. Klier, S. Weber, J. Jonuscheit, and G. von Freymann, "Terahertz time-domain technology for thickness determination of industrial relevant multi-layer coatings," Infrared, Millimeter, and Terahertz waves (IRMMW-THz), 2016 41st International Conference on (2016).

4. H. Merbold, D. J. H. C. Maas, and J. L. M. van Mechelen, "Multiparameter sensing of paper sheets using terahertz time-domain spectroscopy: Caliper, fiber orientation, moisture, and the role of spatial inhomogeneity," SENSORS, 2016 IEEE, 1-3 (2016).

5. S. Weber, J. Klier, F. Ellrich, S. Paustian, N. Guettler, O. Tiedje, J. Jonuscheit, and G. von Freymann, "Thickness determination of wet coatings using self-calibration method," Infrared, Millimeter, and Terahertz Waves (IRMMWTHz), 2017 42nd International Conference on (2017).

6. T. Yasui, T. Yasuda, K.-i. Sawanaka, and T. Araki, "Terahertz paintmeter for noncontact monitoring of thickness and drying progress in paint film,” Appl. Opt. 44, 6849-6856 (2005).

7. L. Duvillaret, F. Garet, JL. Coutaz, "Highly precise determination of optical constants and sample thickness in terahertz time-domain spectroscopy," Applied optics 38 (2), 409-415 (1999).

8. D. Molter, M. Trierweiler, F. Ellrich, J. Jonuscheit, and G. von Freymann, "Interferometry-aided terahertz time-domain spectroscopy," Opt. Express 25 (7), 7547-7558 (2017).

9. T. Pfeiffer, S. Weber, J. Klier, S. Bachtler, D. Molter, J. Jonuscheit, G.v. Freymann, "Terahertz thickness determination with interferometric vibration correction for industrial applications," Optics Express 26 (10), 12558-12568 (2018).

10. P. Uhd Jepsen, R. H. Jacobsen, and SR. Keiding, "Generation and detection of terahertz pulses from biased semiconductor antennas," JOSA B 13 (11), 2424-2436 (1996).

11. D. Grischkowsky et al., "Far-infrared time-domain spectroscopy with terahertz beams of dielectrics and semiconductors," JOSA B 7(10), 2006-2015 (1990).

12. Z. Piao et al., "Carrier Dynamics and Terahertz Radiation in Photoconductive Antennas," Japanese Journal of Applied Physics 39.1R, 96 (2000).

13. S. Krimi, J. Klier, F. Ellrich, J. Jonuscheit, R. Urbansky, R. Beigang, and G. von Freymann, "An evolutionary algorithm based approach to improve the limits of minimum thickness measurements of multilayered automotive paints," Infrared, Millimeter, and Terahertz waves (IRMMW-THz), 2015 40th International Conference on (2015).

14. S. Krimi, J. Klier, J. Jonuscheit, G. von Freymann, R. Urbansky, and R. Beigang, "Highly accurate thickness measurement of multi-layered automotive paints using terahertz technology," Appl. Phys. Lett. 109, 021105 (2016).

15. S. Krimi, G. Torosyan, and R. Beigang, "Advanced GPU-based terahertz approach for in-line multilayer thickness measurements," IEEE Journal of Selected Topics in Quantum Electronics 23 (4), 1-12 (2016).

16. Van Mechelen, JLM ; Kuzmenko, AB ; Merbold, H, "Stratified dispersive model for material characterization using terahertz time-domain spectroscopy," Optics letters 39, 3853-3856 (2014).

17. Naftaly, Mira and Dudley, Richard, "Methodologies for determining the dynamic ranges and signal-to-noise ratios of terahertz time-domain spectrometers," Optics letters 348, 1213-1215 (2009).

18. IE. Gordon, LS. Rothman, C. Hill, RV. Kochanov, Y. Tan, PF. Bernath, M. Birk, V. Boudon, A. Campargue, and KV. Chance, "The HITRAN2016 molecular spectroscopic database," Journal of Quantitative Spectroscopy and Radiative Transfer (203), 3-69 (2017).

Publisher's Note Springer Nature remains neutral with regard to jurisdictional claims in published maps and institutional affiliations. 